PROCEEDINGS OF THE

AMERICAN MATHEMATICAL SOCIETY

Volume 131, Number 9, Pages 2639-2640

S 0002-9939(03)07091-6

Article electronically published on April 1, 2003

\title{
A SIMPLE PROOF OF A THEOREM OF BOLLOBÁS AND LEADER
}

\author{
HONG BING YU
}

(Communicated by David E. Rohrlich)

\begin{abstract}
By using Scherk's lemma we give a simple combinatorial proof of a theorem due to Bollobás and Leader. For any sequence of elements of an abelian group of order $k$, calling the sum of $k$ terms of the sequence a $k$-sum, if 0 is not a $k$-sum, then there are at least $r-k+1 k$-sums.
\end{abstract}

In 3 Erdös, Ginzburg and Ziv proved an elegant result in Combinatorial Number Theory: let $a_{1}, \cdots, a_{2 k-1}$ be a sequence of elements of an abelian group $G$ of order $k$. Then some $k$-sum is 0 (in $G$ ), where (here and below) a $t$-sum is a sum of the form $a_{i_{1}}+\cdots+a_{i_{t}}\left(i_{1}<\cdots<i_{t}\right)$.

There are many proofs and refinements of this result in the literature (see for example [1] and [5, Chap. 2]). Recently, Bollobás and Leader [2] established the following interesting result, which clearly implies the Erdös-Ginzburg-Ziv theorem by taking $r=k-1$.

Theorem. Let $G$ be an abelian group of order $k$, and let $r \geq 1$. Let $A=$ $\left\{a_{1}, \cdots, a_{k+r}\right\}$ be a sequence of elements of $G$. Then if 0 is not a $k$-sum, the number of $k$-sums of $A$ is at least $r+1$.

The proof of the theorem given in [2, pp.30-32] is difficult and complicated. In this note we shall present a simple combinatorial proof of this result. Our argument is based upon the following result of Scherk on addition of subsets of an abelian group (see [6] for a short proof; cf. also [4, Theorem 15' of Chap. 1]).

Lemma. Let $B$ and $C$ be two subsets of an abelian group of order $k$. Suppose $0 \in B \cap C$ and suppose that if $b+c=0$ with $b \in B$ and $c \in C$, then $b=c=0$. Then $|B+C| \geq \min (k,|B|+|C|-1)$, where (here and below) $B+C$ consists of all the elements $b+c$ with $b \in B$ and $c \in C$.

Now we prove the theorem. Translating (which does not affect $k$-sums), we may assume that 0 is the most repeated value in $A$. Let $L$ be the subsequence of all 0 's in $A$, and let $l$ be the cardinality of $L$. Then $l \leq k-1$. Clearly, we can take a subsequence $S$ of $A \backslash L$ summing to 0 with maximal cardinality $s$ and with $s \leq k-1$ ( $S$ may be empty). Then

$$
l+s \leq k-1
$$

Received by the editors December 5, 2001.

2000 Mathematics Subject Classification. Primary 11B50, 20D60.

The author was supported by the National Natural Science Foundation of China.

(C)2003 American Mathematical Society 
for, otherwise, $S$ with $k-s$ 0's of $L$ added would be a subsequence of $A$ with length $k$ summing to 0 . Hence the cardinality of $A \backslash L \cup S$ is at least $r+1$. Taking a subsequence $T$ of $A \backslash L \cup S$ with length $r$, and denoting by $h$ the greatest number of times that any element occurs in $T$, then $h \leq l$ by the definition of $l$. We partition the elements of the sequence $T$ into $h$ sets (not sequences) $X_{1}, \cdots, X_{h}$, and let $X_{i}^{\prime}=X_{i} \cup\{0\}(i=1, \cdots, h)$. We note that $0 \notin T$ and that $T$ has no $j$-sum being 0 for any $1<j \leq h$. For, otherwise, adding such a $j$-terms sequence to $S$ would give us a subsequence $S^{\prime}$ of $A \backslash L$ summing to 0 , but $s^{\prime}$, the cardinality of $S^{\prime}$, satisfies that $s<s^{\prime} \leq h+s \leq l+s \leq k-1$ (by $(*)$ ), contradicting the choice of $S$. Then, by repeatedly applying Scherk's lemma, we have

$$
\left|X_{1}^{\prime}+\cdots+X_{h}^{\prime}\right| \geq\left|X_{1}\right|+\cdots+\left|X_{h}\right|+1=r+1
$$

(recalling that the cardinality of $T$ is $r$ ). In other words, $T$ with $h$ zeros from $L$ appended has at least $r+1 h$-sums. By adding the remaining $k+r-(r+h)$ elements of $A$ to each of these $h$-sums, we obtain at least $r+1 k$-sums of $A$. The proof of the theorem is complete.

\section{ACKNOWLEDGMENT}

The author is grateful to the referee for the suggestion in the revision of the paper.

\section{REFERENCES}

1. N. Alon and M. Dubiner, Zero-sum sets of prescribed size, in: "Combinatorics, Paul Erdös is Eighty", János Bolyai Math. Soc., 1993, pp.33-50. MR 94j:11016

2. B. Bollobás and I. Leader, The number of $k$-sums modulo $k$, J. Number Theory 78 (1999), 27-35. MR 2000i:11036

3. P. Erdös, A. Ginzburg, and A. Ziv, Theorem in the additive number theory, Bull. Res. Council Israel(F) 10 (1961), 41-43.

4. H. Halberstam and K. F. Roth, "Sequences", Vol.I, Oxford Univ. Press, 1966. MR 35:1565

5. M. B. Nathanson, "Additive Number Theory. Inverse Problems and the Geometry of Sumsets", Volume 165 of Graduate Texts in Mathematics, Springer-Verlag, 1996. MR 98f:11011

6. P. Scherk, Solution to Problem 4466, Amer. Math. Monthly 62 (1955), 46-47.

Department of Mathematics, University of Science and Technology of China, Hefei 230026, Anhui, People's Republic of China

E-mail address: yuhb@ustc.edu.cn 